\title{
Fibroin protein/chitosan scaffolds and bone marrow mesenchymal stem cells culture in vitro
}

\author{
J. Deng ${ }^{1}$, R.F. She ${ }^{2}$, W.L. Huang ${ }^{1}$, C. Yuan ${ }^{1}$ and G. Mo ${ }^{1}$ \\ ${ }^{1}$ Department of Orthopedics, Third Affiliated Hospital of Zunyi Medical College, \\ Zunyi, Guizhou, China \\ 2Department of Orthopedics, Guizhou Provincial People's Hospital, \\ Guiyang, Guizhou, China \\ Corresponding author: J. Deng \\ E-mail: dj30666@126.com
}

Genet. Mol. Res. 13 (3): 5745-5753 (2014)

Received May 8, 2013

Accepted November 12, 2013

Published July 29, 2014

DOI http://dx.doi.org/10.4238/2014.July.29.1

\begin{abstract}
A previous experiment demonstrated that fibroin protein and chitosan mixed in proper proportion presented good physical and chemical properties and biological characteristics, which can make up for their respective disadvantages. To observe the growth of bone marrow mesenchymal stem cells (BMSCs) on these fibroin protein/ chitosan 3D scaffolds, induced rabbit BMSCs were seeded on fibroin protein/chitosan scaffolds. The cell adhesion rate was measured, and cell growth was observed under an inverted microscope and a scanning electron microscope. The cell adhesion rate increased with time. The inverted microscope observations showed that the cells on fibroin protein/chitosan scaffolds could not be seen clearly. As time passed, the number of cells around the stent increased and some cells stretched inside the scaffolds. Electron microscopy showed active cell growth and normal proliferation, and the granular and filamentous matrix substances could be seen around cells. The microfilaments of cell and scaffold materials were tightly connected. The cells not
\end{abstract}


only grew on the surface of the adherent material, but also stretched inside of the materials. These results indicated that the fibroin protein/ chitosan mixed scaffolds have good biocompatibility.

Key words: Fibroin protein; Chitosan; Scaffold; Biomaterials; Tissue engineering; Bone marrow mesenchymal stem cells

\section{INTRODUCTION}

The two core materials of cartilage tissue engineering are carrier scaffolds and seed cells (Casper et al., 2010; Bhardwaj et al., 2011). Establishing good construction between the carrier scaffolds and the seed cells is a critical factor for efficient engineering (Tritz-Schiavi et al., 2010). Mesenchymal stem cells in the bone marrow (BMSCs) have multiple differentiation ability, can be cultured in vitro to proliferate and split stably, and can also differentiate toward chondrocytes in a specific induced environment (Frosch et al., 2006; Yang et al., 2008). Silk fibroin and chitosan are natural materials that have good biocompatibilities (Wang et al., 2010a; Li et al., 2011). This experiment employed silk fibroin/chitosan scaffolds that were previously shown to have good physical and chemical properties and to confer a suitable environment for cell survival. The induced chondrocyte differentiated BMSCs were seeded in the silk fibroin/chitosan scaffolds, cell growth was observed, and the compatibility of the cell materials was analyzed to explore the feasibility of using silk fibroin/chitosan scaffolds as cell carriers for cartilage tissue repair.

\section{MATERIAL AND METHODS}

\section{Experimental design}

The mixed culture experiments were performed with scaffolds and cells in vitro in the central laboratory of Zunyi Medical College between December 2010 and August 2011.

\section{Experimental animals}

Twelve male and female New Zealand white rabbits of clean grade were provided by Kunming Medical College. Rabbits were 2.5 months of age with an average body mass of 3.0 $\pm 0.2 \mathrm{~kg}$. The license numbers for laboratory animal production and use were SCXK (Yunnan) 20050008 and SYXK (Yunnan) 20050004, and the animals were fed in a single cage. The disposal of animals in the experimental process was in line with the guidance on the care of laboratory animals released by the National Technical Ministry (2006).

\section{Silk fibroin/chitosan scaffold materials preparation}

The prepared $3 \mathrm{~mm}$ tall, $5 \mathrm{~mm}$ in diameter cylindrical scaffolds were ultraviolet (UV)irradiated for $40 \mathrm{~min}$ and immersed in $75 \%$ ethanol for $30 \mathrm{~min}$. They were taken out, washed several times with phosphate-buffered saline, and then UV-irradiated for $30 \mathrm{~min}$. Then they were immersed in $10 \%$ fetal bovine serum medium for $24 \mathrm{~h}$ for further use. 


\section{Rabbit BMSC isolation, purification, culture, amplification, induction, and identification}

To separate and purify BMSCs, the rabbits' femur bone marrow underwent gradient centrifugation and the adherent growth method (Redzić et al. 2010). When the cells were amplified and cultured to the $3 \mathrm{rd}$ generation, they were added to the induced solution $(10 \mu \mathrm{g} / \mathrm{L}$ transforming growth factor- $\beta 1,50 \mu \mathrm{g} / \mathrm{L}$ ascorbic acid, $40 \mu \mathrm{g} / \mathrm{L}$ dexamethasone, and high glucose Dulbecco's modified Eagle's medium) for induced culture for 14 days. BMSCs were detected using collagen type II and glycosaminoglycan indicators to determine whether or not they were chondrocyte differentiated.

\section{Vaccinated composition of the cell and scaffold materials}

After 14 days of induction, the 3rd generation BMSCs were digested, centrifuged, and adjusted to obtain a cell suspension at a $1.3 \times 10^{8}$ cells/L concentration. They were then seeded in silk fibroin/chitosan scaffold culture plates as the experimental group, and cells inoculated in pure culture plates were taken as the control group. All cells were cultured in a $\mathrm{CO}_{2}$ incubator. At 2, 4, and $6 \mathrm{~h}$ after inoculation, the cells were counted to calculate the adhesion rate between the cells and scaffolds.

After 14 days of induction, the 3rd generation BMSCs were digested, centrifuged, and adjusted to obtain a cell suspension at a $1.4 \times 10^{11}$ cells/Lconcentration. The whole medium soaked scaffolds were put into 24 -well plates, and the liquid in the scaffolds was sucked out. A $90-\mu \mathrm{L}$ cell suspension was seeded on the scaffolds in the cell incubator for $4 \mathrm{~h}$ of culture. The scaffolds were then taken out and flipped to another side with $70 \mu \mathrm{L}$ cell suspension on the surface. They were placed in the culture incubator for $3 \mathrm{~h}$. The complete medium was added to immerse the scaffolds. They were placed in a cell incubator and the culture medium was changed every 2 days.

\section{Inverted microscope observations}

The growing conditions and morphological changes of the cells in the scaffolds were observed daily under an inverted microscope.

\section{Scanning electron microscope observations}

At 1,3 , and 5 days after cell inoculation, one specimen was randomly taken out and observed, respectively. The specimen was fixed with $2.5 \%$ glutaraldehyde, dehydrated with gradient ethanol, dried at critical points, sprayed gold with the ion-sputtering instrument, and observed with scanning electron microscopy.

\section{Main outcomes measurements}

The growth and adhesion of BMSCs on silk fibroin/chitosan scaffolds were recorded.

\section{Statistical analysis}

The SPSS13.0 statistical software was used to process and analyze the results. Data 
are reported as means $\pm \mathrm{SD}$ and the two groups were compared using the Student's $t$-test. $\mathrm{P}<$ 0.05 represented statistically significant differences.

\section{RESULTS}

\section{Culture and induction of isolated BMSCs}

At $48 \mathrm{~h}$ in the primary generation, the cells showed the short and rod shape that grew adherent to the wall, the quantity of the cells was limited, and many blood cells were floating in the medium, as shown in Figure 1A. The first generation cells showed the fusiform and irregular shape, the cells grew well, and the number of blood cells floating in the medium significantly decreased as shown in Figure 1B. The second generation cells showed the fusiform shape and grew adherent to the wall, the cell proliferation was active, and the cells grew rapidly as shown in Figure 1C. The morphology of third generation cells was consistent with that of the second generation cells, showing the fusiform or irregular shape, and the proliferation was fast and the cells grew well as shown in Figure 1D. The collagen II histochemical detection (Figure 2) and the toluidine blue detecting cosaminoglycans (Figure 3 ) were both positive in the third generation cells.

A

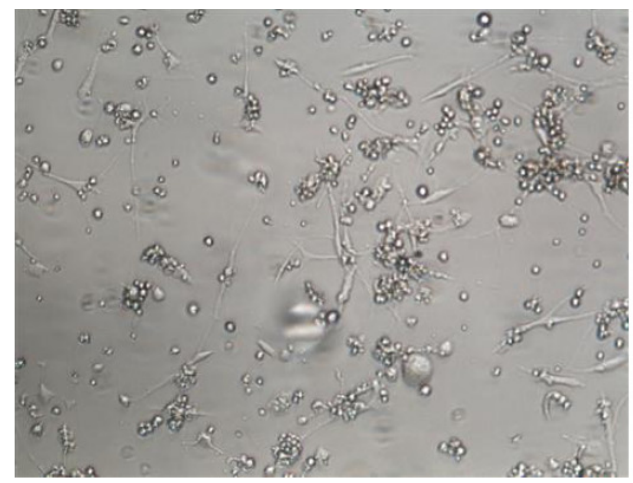

C

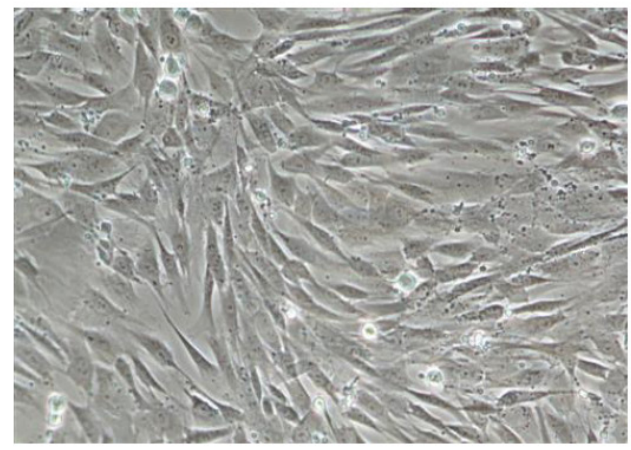

B

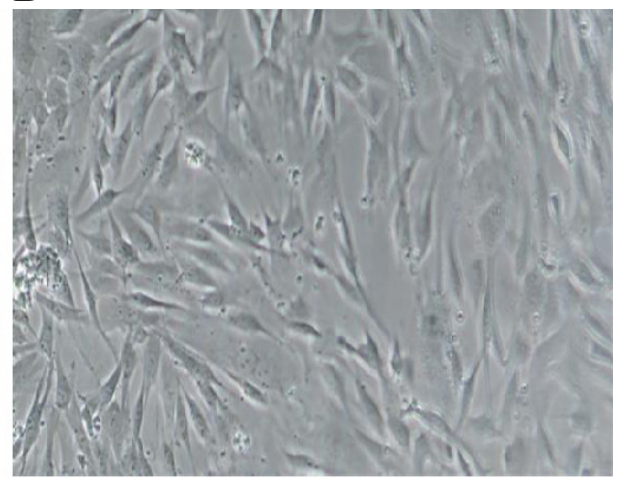

D

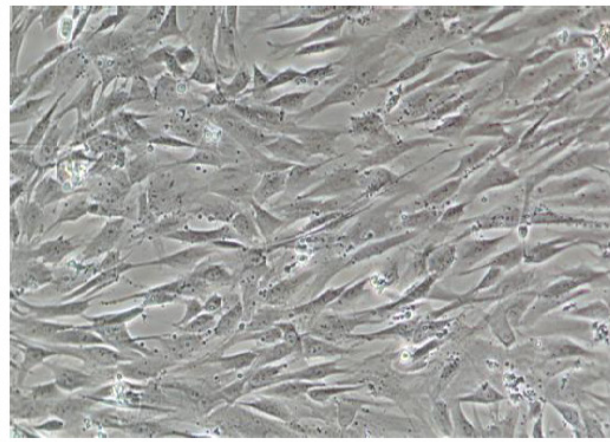

Figure 1. A. Primary cells for 48 hours (100X): B. First generation cells (100X); C. Second generation cell (100X): D. Third generation cell (100X). 


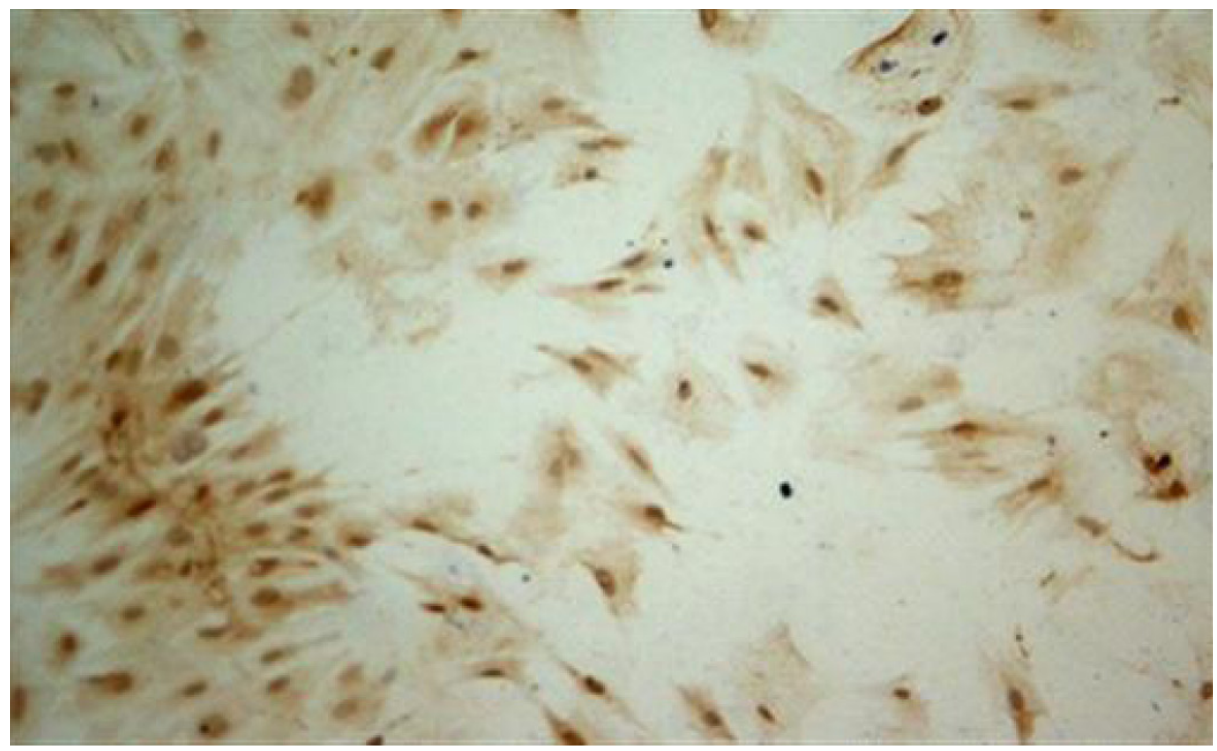

Figure 2. Third generation cells after induction of type II collagen immunohistochemical (100X).

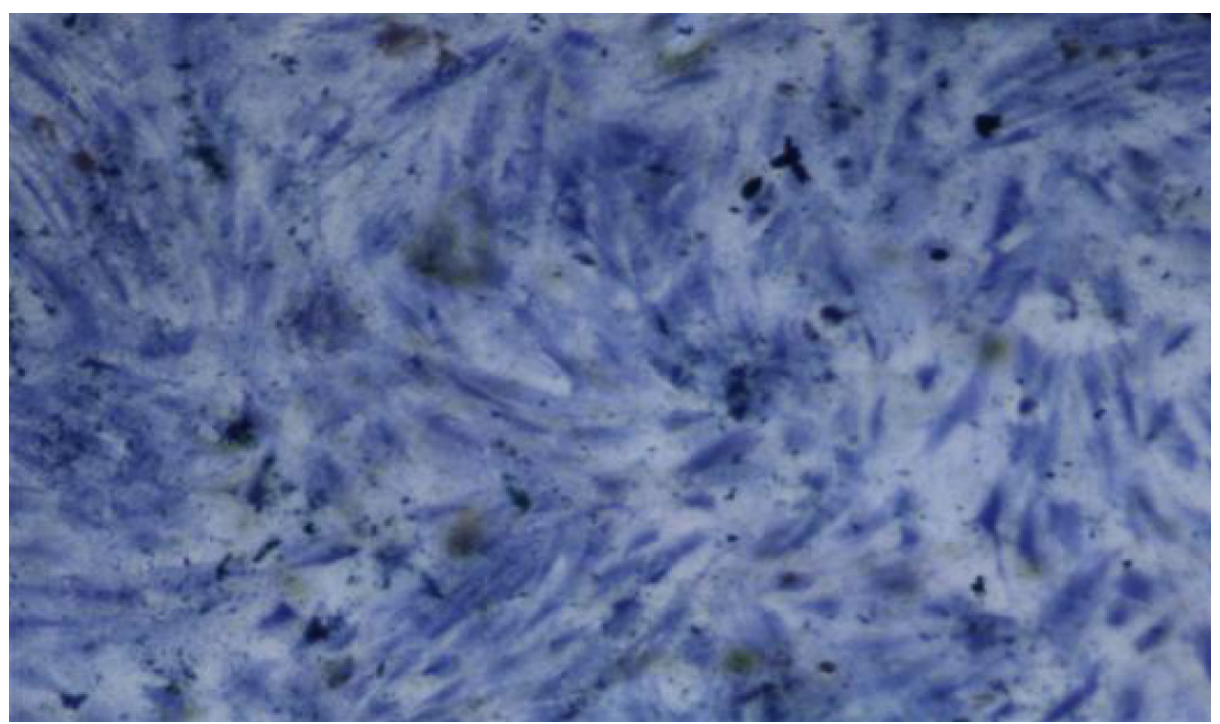

Figure 3. Third generation cells after induction of toluidine blue staining (100X).

\section{Cells and scaffolds adhesion rate detection}

The experimental results showed that the cell adhesion increased with time, and was significantly higher in the experimental group compared to the control group $(\mathrm{P}<0.05)$ (Table 1). 
Table 1. Cell adhesion rate of SF-CS scaffold (means \pm SD).

\begin{tabular}{lccc}
\hline Group & \multicolumn{3}{c}{ Time $(\mathrm{h})$} \\
\cline { 2 - 4 } & 2 & 4 & 6 \\
\hline Experiment & $65.44 \pm 0.99$ & $80.27 \pm 1.62$ & $88.81 \pm 1.99$ \\
Control & $53.05 \pm 1.58$ & $64.38 \pm 1.78^{\star}$ & $77.27 \pm 2.56^{\mathbf{\Delta}}$ \\
\hline
\end{tabular}

$\triangle$ Meant that there was statistically significant difference when compared with the experimental group $(\mathrm{P}<0.05)$.

\section{BMSCs growth on the scaffolds under the inverted microscope}

Under the inverted microscope, the scaffolds were not translucent and the cells could not be seen clearly. A large number of round cell suspensions could be seen around the scaffolds surrounding the medium during the inoculation. One day later, the short spindle cells were adherent to the wall at the bottom of the plates around the scaffolds (Figure 4A). Three days later, the number of cells around the scaffolds increased, which were closer to the scaffolds, and a larger number of the cells showed the long spindle shape. Five days later, the cell density increased significantly around the scaffolds, which were closer to the edge of the scaffolds, there was a greater density of cells, the cells tended to grow inside the scaffolds, and the cells' morphology was long and spindle shaped (Figure 4B).

A

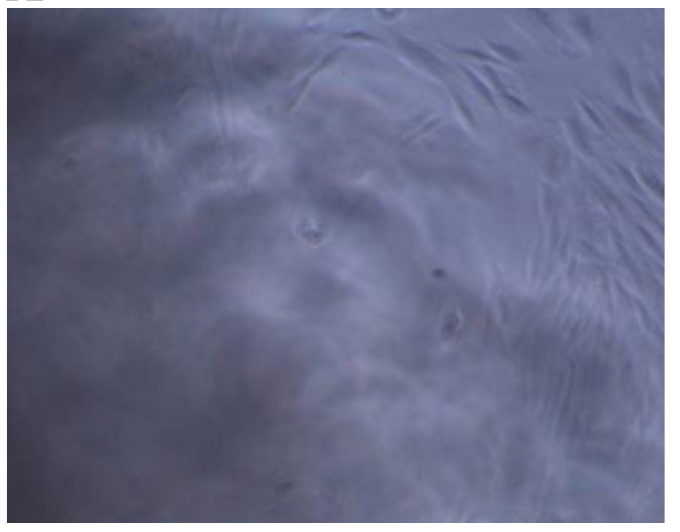

B

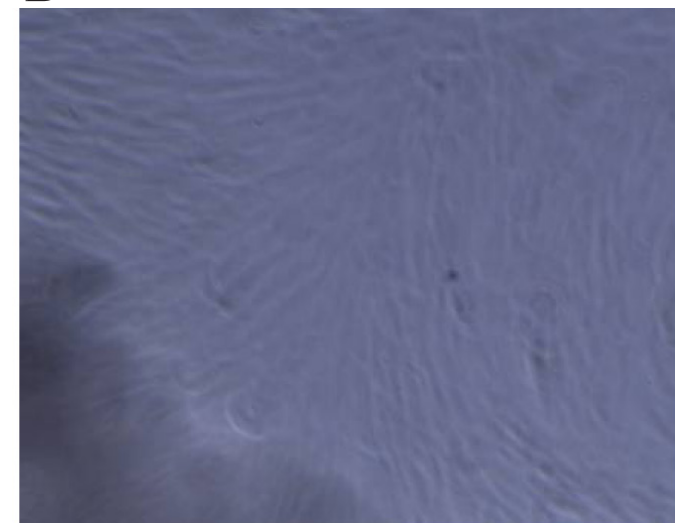

Figure 4. Growth of the cells which were seeded in the SF-CS scaffolds at 1 day and 5 days under the inverted microscope respectively. A. Cells on the scaffold 1 day was observed under inverted microscope (100X); B. Cells on the scaffolds 5 days was observed under inverted microscope (100X).

\section{BMSCs growth on the scaffolds under the electron microscope}

One day after inoculation, electron microscopy revealed that there were scattered round cells attached to the silk fibroin/chitosan scaffolds, there were a lot of bumps on the surface of the cells, the microfilaments and scaffolds materials were connected, and there were a small number of particles observed around the cells (Figure 5A). Three days later, a large number of cells adhered to the surface and pore of scaffolds. The cell growth was exuberant 
and the proliferation was active. The granular and filamentous matrix substances could be seen surrounding cells. The microfilaments of cells were closely connected with the scaffold materials (Figure 5B). Five days later, a large number of cells could be seen adhered to the surface and pore of scaffolds, which outnumbered other cells. The cell growth and proliferation were active, and the cell morphology changed from round to long spindle or oval. They grew from the cell surface to the scaffolds pores. The microfilaments allowed the cells to suspend in the pores of the scaffolds (Figure 5C and D).

A

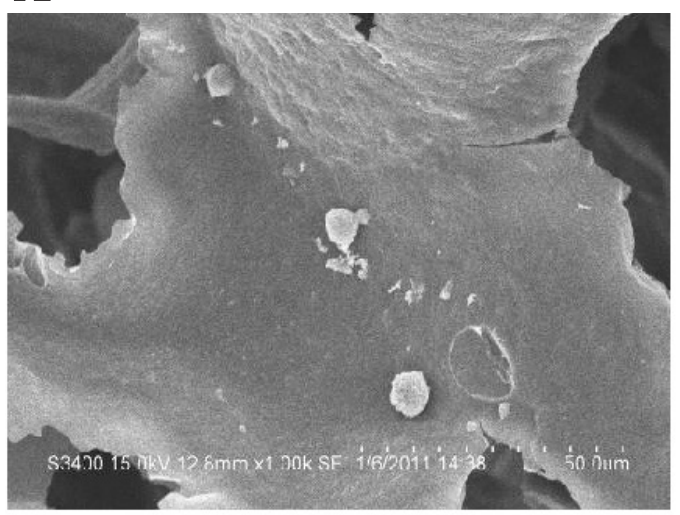

C

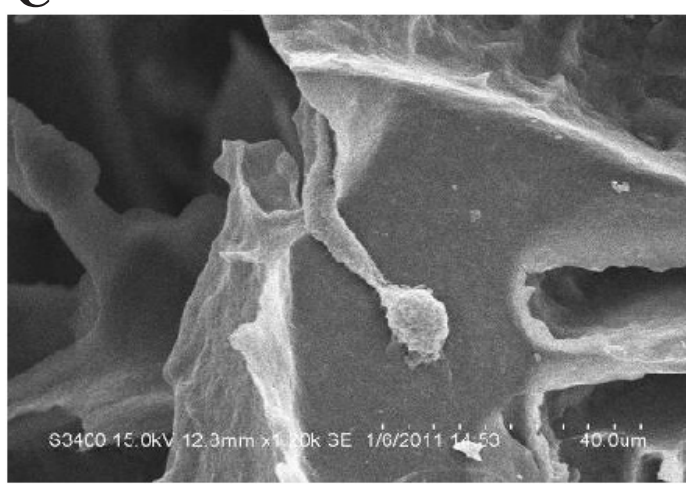

B

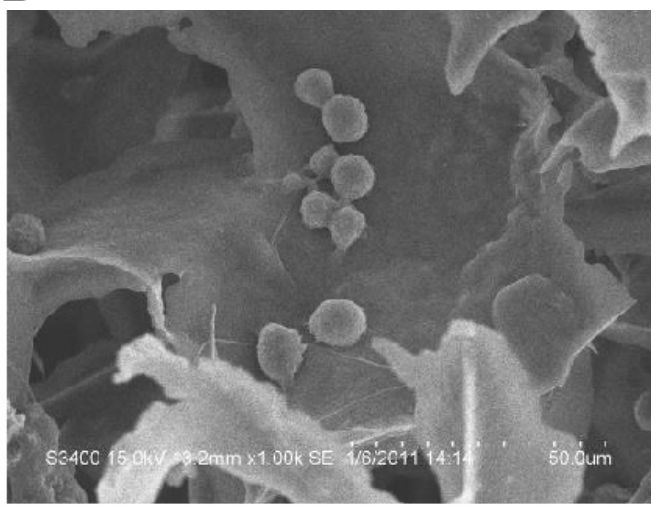

D

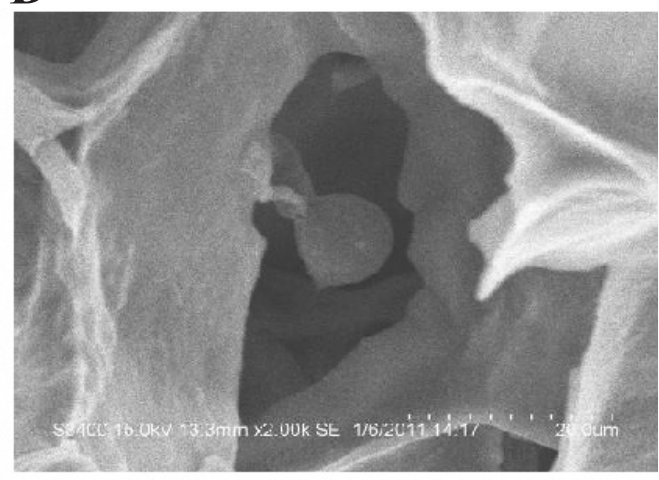

Figure 5. A. Cells on the scaffold 1 day were observed by scanning electron microscope $(1.00 \mathrm{kX})$; B. Cells on the scaffold 3 days were observed by scanning electron microscope $(1.00 \mathrm{kX}) ; \mathbf{C}$. Cells on the scaffold 5 days were observed by scanning electron microscope $(1.20 \mathrm{kX})$; D. Cells on the scaffold 5 days were observed by scanning electron microscope $(2.00 \mathrm{kX})$. Note: A and B showed the growth of the cells which were seeded in the SF-CS scaffolds at 1 day and 3 days under the electron microscopy respectively. $\mathrm{C}$ and $\mathrm{D}$ showed the growth of the cells which were seeded in the SF-CS scaffolds at 5 days under the electron microscopy respectively. A. $1.00 \mathrm{kX}, \mathbf{B}$. $1.00 \mathrm{kX}$, C. $2.00 \mathrm{kX}$, D. $2.00 \mathrm{kX}$.

\section{DISCUSSION}

The silk fibroin protein extracted from silk has advantages of non-toxicity, non-stimu- 
lation, good biocompatibility, cell adsorption, and biodegradation (Redzić et al., 2010). It has broad application prospects in the biomedical field such as surgery sutures, wound protection materials, artificial blood vessels, artificial tendons, drug sustained-release, and anti-clotting substances (Jiang et al., 2006; Rockwood et al., 2011). Chitosan is the deacetylated product of chitin, and has advantages of non-toxicity, odorlessness, absorbance, easy processing, and good biological features (Hines and Kaplan, 2011). Chitosan has been widely used for biomedical material engineering such as artificial skin, absorbable sutures, hemostatic sponges, anti-adhesion agents, cartilage repair, and sustained-released drug membranes (Shi et al., 2006; Wang et al., 2010a). The fibroin protein and chitosan are also widely applied in tissue engineering scaffolds; however, when used alone some shortcomings are apparent. Therefore, the two were mixed to make scaffolds for soft tissue repair, and positive research outcomes were achieved (Silva et al., 2008; Gunbeyaz et al., 2010). In previous experiments, our research team found that mixing fibroin protein and chitosan in the right proportions could make up for their individual disadvantages. The physical and chemical properties and biological characteristics of the scaffolds may meet the cartilage tissue engineering requirements and show no cytotoxicity. Here, through the observation of the growth of the cells on the scaffolds, we explored the feasibility of this scaffold as a seed cell carrier.

In this study, a purely physical detection method was adapted to detect the cells and scaffolds building, which had the advantage of objectivity, sensitivity, and reproducibility. Furthermore, the experiment was performed in vitro to exclude the impact of complex factors in the body, thus reflecting the adhesion of cells and scaffold materials more directly. The adhesion of cells and scaffold materials increased with time, and the cell adhesion rate in the experimental group was higher than that in the control group. The inverted microscope observations showed that the cell numbers increased at the edge of the scaffolds every day, moving increasingly closer to the scaffolds. There was a tendency for cells to move deeper in scaffolds over time. The experimental results showed that this scaffold had good cell compatibility and could promote the proliferation of cells.

Scanning electron microscopy is widely used in observations of scaffold microstructure and growth of cells in the scaffolds. It has the advantages of simple preparation procedures, strong intuitivism, and can also reflect cell growth on the scaffolds clearly and accurately (Altman et al., 2009). In this study, the fibroin protein/chitosan composite and induced stem cells were combined and cultured in vitro. Scanning electron microscope observations revealed that the cells grew actively on scaffolds, the cells' proliferation was normal, and the cell and the scaffold materials were well connected. There were a large number of cells observed in the scaffold pores, suggesting that the scaffold materials could provide a suitable environment for the cells' proliferation. The cells not only grew on the materials' surfaces, but also extended into the materials. We also observed cells in the pores, which indicated that the scaffold aperture size was suitable for providing the space for cell growth and efficient channels for nutrients to reach the cells. In addition, we observed granular and filamentous matrix materials in surrounding cells, which could be the polysaccharides or proteins secreted by the cartilage cells that the stem cells transformed into. These results showed that the scaffold material had good biocompatibility that could provide a suitable three-dimensional environment for cell growth, and might also promote cell proliferation and differentiation.

The experimental results demonstrated that fibroin protein/chitosan scaffolds would serve as a good implanter. This is most likely due to the fact that the fibroin protein and chitosan 
are natural materials with good cell compatibility as scaffolds, so that mixing the two with a physical method retains their respective advantages. Chitosan has a hydrophilic chemical group and is the only positively charged natural material (Muzio et al., 2010) that can provide good adhesion with negatively charged cells. Chitosan also has antibacterial effects that can provide a sterile environment for cell growth (Aimin et al., 1999; Makhlof et al., 2010; Wang et al., 2011). Previous experiments confirmed the strong absorbent ability of the fibroin protein/chitosan scaffolds, allowing cells to be absorbed in the scaffolds during the vaccination.

Because the results of the study were based only on in vitro experiments, the efficacy of these scaffolds for induced stem cells to repair cartilage defects in organisms remains to be the investigated.

\section{REFERENCES}

Aimin C, Chunlin H, Juliang B, Tinyin Z, et al. (1999). Antibiotic loaded chitosan bar. An in vitro, in vivo study of a possible treatment for osteomyelitis. Clin. Orthop. Relat. Res. 239-247.

Altman AM, Yan Y, Matthias N, Bai X, et al. (2009). IFATS collection: Human adipose-derived stem cells seeded on a silk fibroin-chitosan scaffold enhance wound repair in a murine soft tissue injury model. Stem Cells 27: 250-258

Bhardwaj N, Nguyen QT, Chen AC, Kaplan DL, et al. (2011). Potential of 3-D tissue constructs engineered from bovine chondrocytes/silk fibroin-chitosan for in vitro cartilage tissue engineering. Biomaterials 32: 5773-5781.

Casper ME, Fitzsimmons JS, Stone JJ, Meza AO, et al. (2010). Tissue engineering of cartilage using poly-epsiloncaprolactone nanofiber scaffolds seeded in vivo with periosteal cells. Osteoarthritis Cartilage 18: 981-991.

Frosch KH, Drengk A, Krause P, Viereck V, et al. (2006). Stem cell-coated titanium implants for the partial joint resurfacing of the knee. Biomaterials 27: 2542-2549.

Gunbeyaz M, Faraji A, Ozkul A, Purali N, et al. (2010). Chitosan based delivery systems for mucosal immunization against bovine herpesvirus 1 (BHV-1). Eur. J. Pharm. Sci. 41: 531-545.

Hines DJ and Kaplan DL (2011). Mechanisms of controlled release from silk fibroin films. Biomacromolecules 12: 804-812.

Jiang Y, Chen H, Zhou W, Hua J, et al. (2006). Research on preparation of silk fibroin and its biocompatibility with rat bone marrow mesenchymal stem cells. Sheng Wu Yi Xue Gong. Cheng Xue Za Zhi 23: 560-564.

Li XL, Mu CZ and Ma YS (2011). Collagen - chitosan scaffolds with mesenchymal stem cells histocompatibility. Zhongguo Zuzhi Gongcheng Yanjiu yu Linchuang Kangfu 15: 1377-1379.

Makhlof A, Werle M, Tozuka Y and Takeuchi H (2010). Nanoparticles of glycol chitosan and its thiolated derivative significantly improved the pulmonary delivery of calcitonin. Int. J. Pharm. 397: 92-95.

Muzio G, Verne E, Canuto RA, Martinasso G, et al. (2010). Shock waves induce activity of human osteoblast-like cells in bioactive scaffolds. J. Trauma 68: 1439-1444.

Redzić A, Smajilagić A, Aljicević M and Berberović L (2010). In vivo osteoinductive effect and in vitro isolation and cultivation bone marrow mesenchymal stem cells. Coll. Antropol. 34: 1405-1409.

Rockwood DN, Gil ES, Park SH, Kluge JA, et al. (2011). Ingrowth of human mesenchymal stem cells into porous silk particle reinforced silk composite scaffolds: An in vitro study. Acta Biomater. 7: 144-151.

Shi C, Zhu Y, Ran X, Wang M, et al. (2006). Therapeutic potential of chitosan and its derivatives in regenerative medicine. J. Surg. Res. 133: 185-192.

Silva SS, Motta A, Rodrigues MT, Pinheiro AF, et al. (2008). Novel genipin-cross-linked chitosan/silk fibroin sponges for cartilage engineering strategies. Biomacromolecules 9: 2764-2774.

Tritz-Schiavi J, Charif N, Henrionnet C, de IN, et al. (2010). Original approach for cartilage tissue engineering with mesenchymal stem cells. Biomed. Mater. Eng. 20: 167-174.

Wang J, Liu J, Tian H, Li C, et al. (2010a). Comparison study on injectable tissue engineered nucleus pulposus constructed by different cells and chitosan hydrogel. Zhongguo Xiu. Fu Chong. Jian. Wai Ke. Za Zhi 24: 806-810.

Wang J, Hu W, Liu Q and Zhang S (2011). Dual-functional composite with anticoagulant and antibacterial properties based on heparinized silk fibroin and chitosan. Colloids Surf. B Biointerfaces 85: 241-247.

Yang Q, Peng J, Lu S, Sun M, et al. (2008). Fabrication of a novel cartilage acellular matrix scaffold for cartilage tissue engineering. Zhongguo Xiu. Fu Chong. Jian. Wai Ke. Za Zhi 22: 359-363. 